\title{
Fatores relacionados à intensificação do trabalho da enfermagem hospitalar
}

\author{
Factors related to the intensification of hospital nursing work \\ Factores relacionados con la intensificación del trabajo de enfermería hospitalaria
}

Recebido: 05/01/2021 | Revisado: 06/01/2021 | Aceito: 07/01/2021 | Publicado: 09/01/2021

\author{
Éder Luís Arboit \\ ORCID: https://orcid.org/0000-0001-8929-5228 \\ Universidade de Cruz Alta, Brasil \\ E-mail: earboit@unicruz.edu.br \\ Silviamar Camponogara \\ ORCID: https://orcid.org/0000-0001-9342-3683 \\ Universidade Federal de Santa Maria, Brasil \\ E-mail: silviaufsm@yahoo.com.br \\ Etiane de Oliveira Freitas \\ ORCID: https://orcid.org/0000-0002-8589-2524 \\ Universidade Federal de Santa Maria, Brasil \\ E-mail: etiof@yahoo.com.br
}

\begin{abstract}
Resumo
Objetivo: identificar os fatores relacionados à intensificação do trabalho da enfermagem no ambiente hospitalar. Método: estudo de revisão integrativa da literatura, cujo levantamento bibliográfico foi realizado em agosto e setembro de 2019, em seis bases de dados da área da saúde. A amostra esteve constituída por 20 artigos. Realizou-se a sistematização dos dados pela técnica de Análise de Conteúdo Temático. Resultados: a intensificação do trabalho da enfermagem está relacionada aos fatores institucionais/laborais com foco na precarização do trabalho, sub dimensionamento de pessoal com consequente aumento da carga de trabalho, fragilidades nas condições e relações de trabalho, extensão e/ou duplicidade na jornada de trabalho e, neste interim, a realização de varias tarefas de modo simultâneo. Dentre os fatores humanos, destaca-se a ocorrência de alterações físicas e emocionais nos trabalhadores, aumento do estresse, exaustão, fragilidade nas relações e aumento do absenteísmo. Já em relação aos fatores clínicos, estão relacionados com o aumento nas taxas de admissões a altas hospitalares, aumento nas taxas de mortalidade dos pacientes e tempo de permanência hospitalar. Conclusão: evidencia-se que a intensificação do trabalho da enfermagem no ambiente hospitalar advém de fatores institucionais/laborais, fatores humanos e aqueles relacionados com o quadro clínico dos pacientes. O estudo poderá contribuir poderá subsidiar e fomentar discussões sobre a temática, possibilitando à formulação de estratégias direcionadas a saúde do trabalhador e melhoria da qualidade dos cuidados oferecidos aos pacientes.
\end{abstract}

Palavras-chave: Enfermagem; Trabalho; Condições de trabalho; Carga de trabalho; Saúde do trabalhador.

\begin{abstract}
Objective: to identify factors related to the intensification of nursing work in the hospital environment. Method: an integrative literature review study, whose bibliographic survey was carried out in August and September 2019, in six health databases. The sample consisted of 20 articles. Data were systematized using the Thematic Content Analysis technique. Results: the intensification of nursing work is related to institutional / labor factors with a focus on job insecurity, under-dimensioning of staff with a consequent increase in workload, weaknesses in working conditions and relationships, extension and / or duplicity in the workday. work and, in the meantime, the performance of several tasks simultaneously. Among the human factors, the occurrence of physical and emotional changes in the workers, increased stress, exhaustion, fragile relationships and increased absenteeism stand out. In relation to clinical factors, they are related to the increase in the rates of admissions to hospital discharge, increase in the mortality rates of patients and length of hospital stay. Conclusion: it is evident that the intensification of nursing work in the hospital environment comes from institutional / work factors, human factors and those related to the clinical condition of patients. The study may contribute to support and encourage discussions on the theme, enabling the formulation of strategies aimed at workers' health and improving the quality of care offered to patients.
\end{abstract}

Keyword: Nursing; Work; Working conditions; Work load; Worker's health.

\section{Resumem}

Objetivo: identificar factores relacionados con la intensificación del trabajo de enfermería en el ámbito hospitalario. Método: estudio de revisión integrativa de la literatura, cuyo relevamiento bibliográfico se realizó en agosto y septiembre de 2019, en seis bases de datos de salud. La muestra estuvo compuesta por 20 artículos. Los datos fueron sistematizados mediante la técnica de Análisis de Contenido Temático. Resultados: la intensificación del trabajo de 
enfermería se relaciona con factores institucionales / laborales con foco en la precariedad laboral, subdimensionamiento del personal con consecuente aumento de la carga de trabajo, debilidades en las condiciones y relaciones laborales, extensión y / o duplicidad en la jornada laboral trabajo y, mientras tanto, la realización de varias tareas simultáneamente. Entre los factores humanos destacan la ocurrencia de cambios físicos y emocionales en los trabajadores, aumento del estrés, agotamiento, relaciones frágiles y aumento del ausentismo. En relación a los factores clínicos, se relacionan con el aumento de las tasas de ingresos al alta hospitalaria, el aumento de las tasas de mortalidad de los pacientes y la duración de la estancia hospitalaria. Conclusión: es evidente que la intensificación del trabajo de enfermería en el ámbito hospitalario proviene de factores institucionales / laborales, humanos y relacionados con la condición clínica de los pacientes. El estudio puede contribuir a apoyar y fomentar discusiones sobre el tema, permitiendo la formulación de estrategias dirigidas a la salud de los trabajadores y mejorando la calidad de la atención ofrecida a los pacientes.

Palabras clave: Enfermería; Trabajo; Condiciones de trabajo; Carga de trabajo; Salud del trabajador.

\section{Introdução}

O trabalho consiste em uma atividade essencial para a humanidade. De acordo com Marx (2013), o trabalho é um processo de interação dialética entre o ser humano e a natureza e entre os próprios seres humanos, por meio do qual transformam a natureza e a si mesmos. É por meio dele que os indivíduos conquistam novos espaços, obtêm meios de subsistência e realizam trocas imprescindíveis para a sua trajetória individual e coletiva (Rocha, 2020) e, neste contexto, revela-se como ontológico à condição humana.

As modificações no mundo do trabalho são decorrentes da globalização, das mudanças tecnológicas, da incorporação crescente da microeletrônica, da informática, da telemática, da robótica e de aspectos sociais, demográficos e econômicos, as quais trazem implicações diretas e indiretas no cotidiano das pessoas. Dentre estas implicações, destaca-se a predominância maciça do desemprego, acompanhado da ampliação da subutilização da força de trabalho, da precarização nas ocupações (Pochmann, 2020) e acirramento das desigualdades sociais.

No entanto, há aqueles que embora apresentem qualificação, enfrentam dificuldades para nele inserir-se, o que por sua vez, promove um aumento progressivo do mercado informal, instável e precário. Para, além disso, ocorre quem em muitos trabalhadores submetem-se a condições precárias de trabalho e às mais variadas formas de exploração de sua mão de obra (Coutinho; Alves; Santos, 2020).

Neste contexto, ao passo que a atividade laboral encontra-se cada vez mais imersa nas instabilidades do mercado neoliberal (Rocha, 2020), evidencia-se questões relacionadas com diminuição das condições e precarização do trabalho, além das necessidades de maior produtividade. Como consequência, tem-se uma maior intensificação do trabalho, com repercussões significativas na vida pessoal e nos processos laborais. Assim, os trabalhadores, precisam constantemente estar se adequando a fim de atender as necessidades do mercado e dos empregadores.

A intensificação do trabalho, instituída a partir de 1970/1980 é um fenômeno global, que envolve as mais diversas categorias profissionais. Autores como Kubicek, Paškvan e Korunka (2015) a definem como um construto multifacetado, caracterizado pela necessidade de trabalhar mais rápido e cumprir prazos mais apertados, por uma redução do tempo ocioso e pela necessidade de realizar várias tarefas de trabalho, simultaneamente.

Pina e Sortz enfatizam que, a intensificação do trabalho está implicada em práticas de exploração como o prolongamento da jornada, o aumento do ritmo de trabalho e a administração por estresse e, ao mesmo tempo, de expropriação do saber do trabalhador. Esses processos determinam uma pluralidade de agravos à saúde e na manifestação do desgaste e do sofrimento difuso dos trabalhadores (Pina \& Storz, 2014).

Estudo de Souza et al (2017), aponta repercussões do neoliberalismo na organização e no processo de trabalho da equipe de enfermagem no âmbito hospitalar, verificando-se inadequações na estrutura física, nos recursos humanos e materiais, que afetavam a qualidade da assistência. Além disso, a intensificação do trabalho leva ao uso de metodologias laborais menos 
seguras e, consequentemente, traz complicações de ordem musculoesquelética e riscos psicossociais. Isso se dá porque os trabalhadores estão mais propensos a ignorar e bloquear sistemas de segurança para trabalhar mais rápido e manter a produtividade (Bunner, Prem \& Korunka, 2018).

Estudo recente revela que a intensificação e precarização das relações de trabalho, constituem-se em aspectos importantes para se pensar a atuação profissional (Francisco, 2019). Já, em relação ao trabalho do enfermeiro no ambiente hospitalar, Sayin (2018) descreve que os trabalhadores experimentaram a intensificação do trabalho, o que contribuiu para a diminuição da satisfação profissional.

Nesta direção, estudo de Forte (2019) descreve que as causas associadas aos erros de enfermagem estão relacionados à força de trabalho (déficit de profissionais e capacitação, rotatividade, sobrecarga, falta de informação, imprudência, negligência e distração); aos instrumentos de trabalho, semelhança de rótulos/embalagens, armazenamento, falta de identificação e informação de produtos e prescrição médica) e ao objeto de trabalho (particularidades dos pacientes e superlotação) (Forte et al., 2019).

Diante do exposto, depreende-se que o trabalho da enfermagem tem sido influenciado pelo atual contexto de modificações inerentes ao mundo do trabalho contemporâneo. Dentre elas, estudos (quais) apontam que há evidências de intensificação laboral (Selberg 2013); Willis et al. (2015); Zeytinoglu et al. (2015); (2008) e Kubisa (2016).

Contudo, por ser um tema recente e pouco explorado, considera-se relevante conhecer que fatores contribuem para a ocorrência de intensificação do trabalho da enfermagem. Ademais, entende-se que é fundamental identificar os fatores relacionados à intensificação do trabalho, a fim elaborar estratégias para minimizá-los, tendo em vista que as consequências são diretas tanto para a saúde do trabalhador como para a segurança do paciente.

Dessa forma definiu-se como questão norteadora desta revisão integrativa: quais os fatores relacionados à intensificação do trabalho da enfermagem no ambiente hospitalar? O objetivo consiste em identificar os fatores relacionados à intensificação do trabalho da enfermagem no ambiente hospitalar.

\section{Metodologia}

Trata-se de uma Revisão Integrativa de Literatura (RIL), a qual buscou agregar e sumarizar resultados de pesquisas acerca de tema específico, possibilitando a síntese de múltiplos estudos publicados. Além disso, a RIL contribui substancialmente nos melhores resultados para a prática clínica, bem como na identificação de lacunas do conhecimento direcionando o desenvolvimento de novos estudos (Mendes, Silveira \& Galvão, 2019).

$\mathrm{Na}$ operacionalização desta revisão foram seguidas as seguintes etapas indicadas por Hopia; Latvala; Liimatainen (2016): 1) Identificação do tema: a intensificação do trabalho da enfermagem; 2) Elaboração da questão de pesquisa: 3) Estabelecimento de critérios de inclusão: ser artigo de pesquisa na temática disponível online, na íntegra e gratuitamente, em inglês, português ou espanhol; e sem recorte temporal. Como critérios de exclusão destaca-se: artigos sem resumo na base de dados ou incompletos; 4) Definição das informações a serem extraídas dos estudos: referência, ano, procedência, objetivo, abordagem, participantes, principais resultados, e nível de evidência; 5) Avaliação das evidências e análise (categorização): a análise dos dados extraídos foi realizada de forma descritiva, possibilitando a avaliação da força das evidências de acordo com as três pirâmides Fineout-Overholt; Stillwell (2011), a qual leva em conta o tipo de questão clínica do estudo primário; e 6) Discussão e apresentação da síntese do conhecimento evidenciado nos artigos analisados.

O levantamento bibliográfico foi realizado em agosto e setembro de 2019 nas bases de dados: Literatura LatinoAmericana e do Caribe em Ciências da Saúde (LILACS), Base de dados de Enfermagem (BDENF) e Index de psicologia, via Biblioteca Virtual em Saúde (BVS). Também foram realizadas buscas nas bases da US National Library of Medicine National 
Institutesof Health (PUBMED), Sci Verse Scopus (SCOPUS) e Web of Science. O Quadro 1 apresenta as bases de dados e as respectivas estratégias de busca utilizadas em cada uma delas.

Quadro 1 - Fontes de busca, estratégia utilizada e resultados. Santa Maria - RS, 2020.

\begin{tabular}{|c|c|}
\hline BASE / DATA & ESTRATÉGIA DE BUSCA \\
\hline $\begin{array}{c}\text { BVS } \\
25 / 08 / 2019\end{array}$ & $\begin{array}{l}\text { tw:((tw:(enfermagem OR profissionais de enfermagem OR equipe de enfermagem)) AND } \\
\text { (tw:(trabalho OR condições de trabalho OR intensificação do trabalho OR carga de trabalho)) } \\
\text { AND (tw:(hospital OR ambiente hospitalar))) }\end{array}$ \\
\hline $\begin{array}{l}\text { PUBMED } \\
25 / 08 / 2019\end{array}$ & $\begin{array}{l}\text { ("nursing"[MeSH Terms] OR "nurse practitioners"[MeSH Terms]) OR "nursing, team"[MeSH } \\
\text { Terms]) AND (("work"[MeSH Terms] OR working conditions[Title/Abstract]) OR "occupational } \\
\text { health"[MeSH Terms]) OR (intensification[All Fields] AND of work[Title/Abstract]) OR work } \\
\text { demand[Title/Abstract] OR "workload"[MeSH Terms] AND (("hospitals"[MeSH Terms] OR } \\
\text { "hospital"[Title/Abstract]) OR "hospital environment"[Title/Abstract]) }\end{array}$ \\
\hline $\begin{array}{c}\text { SCOPUS } \\
25 / 08 / 2019\end{array}$ & $\begin{array}{l}\text { (TITLE-ABS-KEY ( "nursing" OR "nurse practitioner" OR "nursing, team" ) AND TITLE- } \\
\text { ABS-KEY ( "work" OR "working conditions" OR "occupational health" OR "intensification of } \\
\text { work" OR "Workload" ) AND TITLE-ABS-KEY ( "hospitals" OR "hospital" OR "hospital } \\
\text { environment")) }\end{array}$ \\
\hline $\begin{array}{c}\text { WEB OF SCIENCE } \\
23 / 08 / / 2019\end{array}$ & $\begin{array}{l}\text { (("nursing") OR ("nurse practitioner") OR ("nursing, team")) AND TÓPICO: (("work") OR } \\
\text { ("working conditions") OR ("occupational health") OR ("intensificatión of work") OR } \\
\text { ("Workload")) AND TÓPICO: (("hospitals") OR ("hospital") AND ("hospital environment")) }\end{array}$ \\
\hline
\end{tabular}

Fonte: Autores (2020).

A seleção dos artigos nas bases de dados foi realizada por dois pesquisadores, de modo duplo independente. $\mathrm{Na}$ sequência, realizou-se uma reunião de consenso com os revisores e pesquisador para comparar a seleção realizada por cada revisor. O processo de busca e seleção dos estudos seguiu as recomendações PRISMA e está representado na Figura 1. Para, além disso, também realizou-se a busca manual nas referências dos artigos incluídos no corpus, sendo incorporados cinco artigos que estavam na lista de referências (The Joanna Briggs Institute, 2014). 
Figura 1 - Fluxograma da seleção dos estudos primários. Santa Maria - RS, 2020.

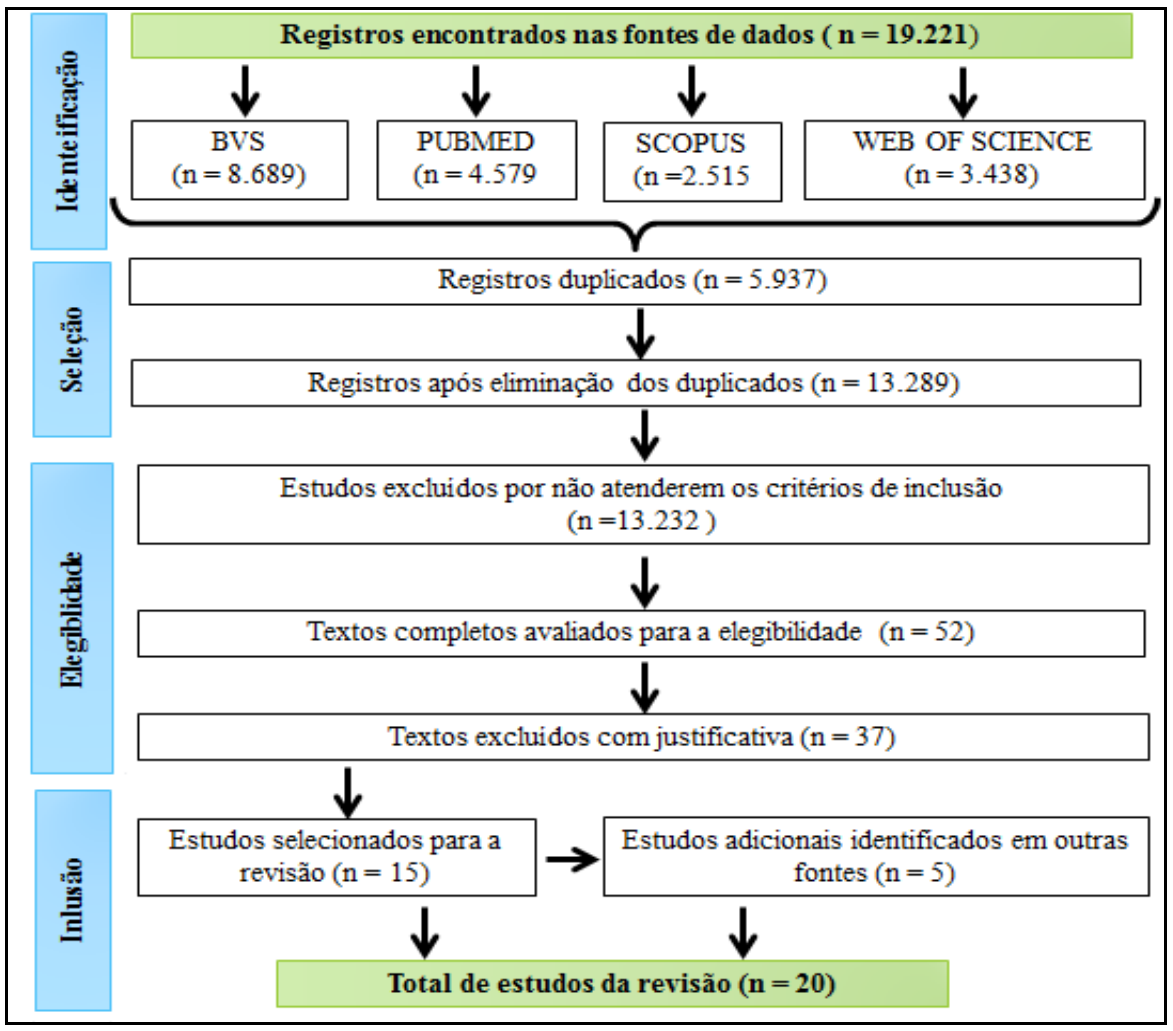

Fonte: adaptado do Preferred Reporting Items for Systematic Review and Meta Analyses (PRISMA).

Conforme apresentado na figura anterior, o corpus de análise está composto por 20 artigos. Para a extração dos dados pertinentes ao estudo e caracterização das produções científicas, utilizou-se um quadro sinóptico, constituído pelas seguintes variáveis: código, primeiro autor, periódico e ano, formação e titulação do primeiro autor, país de origem do estudo, título, objetivo, abordagem metodológica, cenário e população do estudo, principais resultados, base de dados e nível de evidência.

Os artigos foram identificados pela letra "A" de artigo, seguido de uma numeração (A1, A2, A3, e assim sucessivamente). Para a avaliação dos níveis de evidência cientifica, utilizou-se como base, um estudo de Fineout-Overholt; Stillwell (2011), que classifica a força da evidência. Assim, a questão clínica do estudo primário pode ser classificada como sendo de Intervenção/Tratamento, Prognóstico ou Etiologia e de Significado.

\section{Resultados}

Em relação ao ano de publicação, dos 20 artigos incluídos nesta revisão, $30 \%(\mathrm{n}=6)$ foram produzidos no ano de 2018, 20\% ( $(=4)$ em 2017, 15\% ( $n=3)$ em 2013, 10\% ( $=2)$ em 2015 e 2016. Também foram encontrados artigos publicados em 2012, 2008 e 2007 correspondendo a 5\% $(\mathrm{n}=1)$ em cada ano.

Com base na análise dos artigos que compõem o corpus do estudo, constatou-se que, quanto ao local de origem, $70 \%$ $(n=14)$ são internacionais. Em relação ao delineamento metodológico, $65 \%(n=13)$ são de caráter quantitativo, 25\% $(n=5)$ qualitativos, $10 \%(\mathrm{n}=2)$ estudos de abordagem mista. No que se refere aos participantes dos estudos, $70 \%(\mathrm{n}=14)$ foram desenvolvidos com enfermeiros, $25 \%(n=5)$ com a equipe de enfermagem e $5 \%(n=1)$ com base em prontuários de pacientes. O Quadro 2 apresenta a caracterização das produções científicas. 
Quadro 2 - Seleção de informações extraídas dos estudos primários. Santa Maria - RS, 2020.

\begin{tabular}{|c|c|c|c|c|c|}
\hline $\begin{array}{l}\text { Cód. e Base } \\
\text { de dados }\end{array}$ & $\begin{array}{c}1^{\circ} \text { Autor e } \\
\text { Origem do } \\
\text { estudo } \\
\end{array}$ & Periódico e Ano & Título & $\begin{array}{l}\text { Abordagem e } \\
\text { População do } \\
\text { estudo }\end{array}$ & $\begin{array}{l}\text { Nível de } \\
\text { Evidência }\end{array}$ \\
\hline $\begin{array}{l}\text { A1 } \\
\text { Pubmed }\end{array}$ & $\begin{array}{l}\text { Aeschbacher, } \\
\text { R. / Suíça }\end{array}$ & $\begin{array}{l}\text { Hum } \quad \text { Resour } \\
\text { Health. / } 2018 .\end{array}$ & $\begin{array}{l}\text { Institutional effects on nurses' } \\
\text { working conditions: a multi-group } \\
\text { comparison of public and private } \\
\text { non-profit and for-profit healthcare } \\
\text { employers in Switzerland. }\end{array}$ & $\begin{array}{l}\text { Quantitativo / } \\
\text { Enfermeiros }\end{array}$ & $\mathrm{N} 4 * *$ \\
\hline $\mathrm{A} 2$ - BVS & $\begin{array}{l}\text { Carlesi, k. C. I } \\
\text { Chile }\end{array}$ & $\begin{array}{l}\text { Rev. Latino-Am. } \\
\text { Enferm. / } 2017 .\end{array}$ & $\begin{array}{l}\text { Patient Safety Incidents and Nursing } \\
\text { Workload. }\end{array}$ & $\begin{array}{l}\text { Quantitativa / } \\
\text { Prontuários }\end{array}$ & $\mathrm{N} 4 * *$ \\
\hline A3 - BVS & $\begin{array}{l}\text { Aires, K. F. / } \\
\text { Brasil }\end{array}$ & $\begin{array}{l}\text { Rev Enferm } \\
\text { UFPE On Line / } \\
2016 .\end{array}$ & $\begin{array}{l}\text { Contribution of the work load to the } \\
\text { occurrence of medication errors in } \\
\text { nursing. }\end{array}$ & $\begin{array}{l}\text { Quantitativo / } \\
\text { Enfermeiros }\end{array}$ & $\mathrm{N} 4 * *$ \\
\hline $\begin{array}{l}\text { A4 } \\
\text { Pubmed }\end{array}$ & $\begin{array}{l}\text { Fagerström, L. } \\
\text { / Finlândia }\end{array}$ & BMJ / 2018 & $\begin{array}{l}\text { Nursing workload, patient safety } \\
\text { incidents and mortality: an } \\
\text { observational study from Finland. }\end{array}$ & $\begin{array}{l}\text { Quantitativo / } \\
\text { Enfermeiros }\end{array}$ & $\mathrm{N} 4 * *$ \\
\hline $\begin{array}{l}\text { A5 - Web Of } \\
\text { Science }\end{array}$ & $\begin{array}{l}\text { Kim, k. / } \\
\text { Coréia do Sul }\end{array}$ & $\begin{array}{l}\text { Asian Nursing } \\
\text { Research / } 2018 .\end{array}$ & $\begin{array}{l}\text { Exploring the influence of nursing } \\
\text { work environment and patient safety } \\
\text { culture on missed nursing care in } \\
\text { Korea. }\end{array}$ & $\begin{array}{l}\text { Quantitativo / } \\
\text { Enfermeiros }\end{array}$ & $\mathrm{N} 4 * *$ \\
\hline $\begin{array}{l}\text { A6 - Web Of } \\
\text { Science }\end{array}$ & $\begin{array}{l}\text { Barboza, P. C. } \\
\text { / Brasil }\end{array}$ & Rev Rene. / 2018. & $\begin{array}{l}\text { The meaning of work: perspectives of } \\
\text { nursing professionals who work in } \\
\text { clinical units. }\end{array}$ & $\begin{array}{l}\text { Qualitativo / } \\
\text { equipe de } \\
\text { enfermagem } \\
\end{array}$ & $\mathrm{N} 2 * * *$ \\
\hline $\begin{array}{l}\text { A7 - Web Of } \\
\text { Science }\end{array}$ & $\begin{array}{l}\text { Araújo-dos- } \\
\text { Santos, T. / } \\
\text { Brasil. }\end{array}$ & $\begin{array}{l}\text { Rev Esc Enferm } \\
\text { USP. / } 2018 .\end{array}$ & $\begin{array}{l}\text { Job insecurity among nurses, nursing } \\
\text { technicians and nursing aides in } \\
\text { public hospitals. }\end{array}$ & $\begin{array}{l}\text { Quantitativo / } \\
\text { Enfermeiros }\end{array}$ & $\mathrm{N} 4 * *$ \\
\hline $\begin{array}{l}\text { A8 } \\
\text { Pubmed }\end{array}$ & $\begin{array}{l}\text { Semachew, A. } \\
\text { / Etiópia }\end{array}$ & $\begin{array}{l}\text { Human Resources } \\
\text { for Health / } 2017 .\end{array}$ & $\begin{array}{l}\text { Predictors of job satisfaction among } \\
\text { nurses working in ethiopian public } \\
\text { hospitals, 2014: institution-based } \\
\text { cross-sectional study. }\end{array}$ & $\begin{array}{l}\text { Quantitativo / } \\
\text { Enfermeiros }\end{array}$ & $\mathrm{N} 4 * *$ \\
\hline $\begin{array}{l}\text { A9 - Web of } \\
\text { Science }\end{array}$ & $\begin{array}{l}\text { Magalhães, A. } \\
\text { M. M. / Brasil }\end{array}$ & $\begin{array}{l}\text { Rev Esc Enferm } \\
\text { USP / } 2017 .\end{array}$ & $\begin{array}{l}\text { Workloads, strain processes and } \\
\text { sickness absenteeism in nursing. }\end{array}$ & $\begin{array}{l}\text { Quantitativo / } \\
\text { Equipe de } \\
\text { enfermagem }\end{array}$ & $\mathrm{N} 4 * *$ \\
\hline A10 - BVS & $\begin{array}{l}\text { Mininel, V. A. } \\
\text { / Brasil }\end{array}$ & $\begin{array}{l}\text { Rev. Latino-Am. } \\
\text { Enferm. / } 2013 .\end{array}$ & $\begin{array}{l}\text { Workloads, strain processes and } \\
\text { sickness absenteeism in nursing. }\end{array}$ & $\begin{array}{l}\text { Quantitativo / } \\
\text { Equipe de } \\
\text { enfermagem }\end{array}$ & $\mathrm{N} 4 * *$ \\
\hline $\begin{array}{l}\text { A11 } \\
\text { Pubmed }\end{array}$ & $\begin{array}{l}\text { Willis, E. / } \\
\text { Austrália }\end{array}$ & $\begin{array}{l}\text { Labour and } \\
\text { Industry / } 2015 . \\
\end{array}$ & Work intensification as missed care. & $\begin{array}{l}\text { Quantitativo / } \\
\text { Enfermeiros } \\
\end{array}$ & $\mathrm{N} 4 * *$ \\
\hline A12 - BVS & $\begin{array}{l}\text { Magalhães, A. } \\
\text { M. M. / Brasil }\end{array}$ & $\begin{array}{l}\text { Rev. Latino-Am. } \\
\text { Enferm./ } 2013 .\end{array}$ & $\begin{array}{l}\text { Nursing workload and patient safety - } \\
\text { a mixed method study with an } \\
\text { ecological restorative approach. }\end{array}$ & Estudo misto & $\mathrm{N} 2 * * *$ \\
\hline $\begin{array}{l}\text { A13 - Web } \\
\text { Of Science }\end{array}$ & $\begin{array}{l}\text { Doef, M. / } \\
\text { África } \\
\text { Ocidental }\end{array}$ & $\begin{array}{l}\text { Journal of } \\
\text { Clinical Nursing / } \\
2012 \text {. }\end{array}$ & $\begin{array}{l}\text { Job conditions, job satisfaction, } \\
\text { somatic complaints and burnout } \\
\text { among East African nurses. }\end{array}$ & $\begin{array}{l}\text { Quantitativo / } \\
\text { Enfermeiros }\end{array}$ & $\mathrm{N} 4 * *$ \\
\hline $\begin{array}{l}\text { A14 } \\
\text { Scopus }\end{array}$ & $\begin{array}{l}\text { Hart, S. M. I } \\
\text { Suécia }\end{array}$ & $\begin{array}{l}\text { Economic and } \\
\text { Industrial } \\
\text { Democracy } \\
2015 .\end{array}$ & $\begin{array}{l}\text { Understanding nurses' work: } \\
\text { exploring the links between changing } \\
\text { work, labour relations, workload, } \\
\text { stress, retention and recruitment. }\end{array}$ & $\begin{array}{l}\text { Qualitativo / } \\
\text { Enfermeiros }\end{array}$ & $\mathrm{N} 2 * * *$ \\
\hline $\begin{array}{l}\text { A15 } \\
\text { Scopus }\end{array}$ & $\begin{array}{l}\text { Granero, A. / } \\
\text { Espanha }\end{array}$ & $\begin{array}{l}\text { Rev. Latino-Am. } \\
\text { Enferm. / } 2018\end{array}$ & $\begin{array}{l}\text { Labor conditions and the meanings of } \\
\text { nursing work in Barcelona. }\end{array}$ & $\begin{array}{l}\text { Estudo misto / } \\
\text { equipe de } \\
\text { enfermagem }\end{array}$ & $\mathrm{N} 4 * *$ \\
\hline $\begin{array}{l}\text { A16 - } \\
\text { Referências }\end{array}$ & $\begin{array}{l}\text { Selberg, R. / } \\
\text { Suécia }\end{array}$ & $\begin{array}{l}\text { Nordic journal of } \\
\text { working life } \\
\text { studies / 2013. }\end{array}$ & $\begin{array}{l}\text { Nursing in times of neoliberal } \\
\text { change: an ethnographic study of } \\
\text { nurses' experiences of work } \\
\text { intensification. }\end{array}$ & $\begin{array}{l}\text { Etnografia / } \\
\text { Enfermeiros }\end{array}$ & $\mathrm{N} 2 * * *$ \\
\hline $\begin{array}{l}\text { A17 - } \\
\text { Referências }\end{array}$ & $\begin{array}{l}\text { Willis, E. / } \\
\text { Australia }\end{array}$ & $\begin{array}{l}\text { Nursing Inquiry / } \\
2008 .\end{array}$ & $\begin{array}{l}\text { Enterprise bargaining: a case study in } \\
\text { the de-intensification of nursing work } \\
\text { in Australia }\end{array}$ & $\begin{array}{l}\text { Qualitativo / } \\
\text { Equipe de } \\
\text { enfermagem }\end{array}$ & $\mathrm{N} 4 * *$ \\
\hline
\end{tabular}


Research, Society and Development, v. 10, n. 1, e22210111703, 2021

(CC BY 4.0) | ISSN 2525-3409 | DOI: http://dx.doi.org/10.33448/rsd-v10i1.11703

\begin{tabular}{|c|c|c|c|c|c|}
\hline $\begin{array}{l}\text { A18 - } \\
\text { Referências }\end{array}$ & $\begin{array}{l}\text { Zeytinoglu, I. } \\
\text { U. / Canada }\end{array}$ & $\begin{array}{l}\text { Relations } \\
\text { industrielles / } \\
\text { Industrial } \\
\text { Relations / } 2007 .\end{array}$ & $\begin{array}{l}\text { Associations between } \\
\text { intensification, stress and job } \\
\text { satisfaction the case of nurses in } \\
\text { Ontario. }\end{array}$ & $\begin{array}{l}\text { Quantitativo / } \\
\text { Enfermeiros }\end{array}$ & $\mathrm{N} 4 * *$ \\
\hline $\begin{array}{l}\text { A19- } \\
\text { Referências }\end{array}$ & $\begin{array}{l}\text { Willis, E. / } \\
\text { Nova Zelândia }\end{array}$ & $\begin{array}{l}\text { J Nurs Care Qual } \\
\text { / } 2017 .\end{array}$ & $\begin{array}{l}\text { Work Intensification and Quality } \\
\text { Assurance Missed Nursing Care. }\end{array}$ & $\begin{array}{l}\text { Qualitativo / } \\
\text { Enfermeiros }\end{array}$ & $\mathrm{N} 2 * * *$ \\
\hline $\begin{array}{l}\text { A20- } \\
\text { Referências }\end{array}$ & $\begin{array}{lll}\text { Kubisa, J. I } \\
\text { Polônia }\end{array}$ & Emecon / 2016. & $\begin{array}{l}\text { From better job quality to higher- } \\
\text { quality care - Polish nurses' } \\
\text { collective struggle with the public } \\
\text { healthcare system. }\end{array}$ & $\begin{array}{l}\text { Qualitativo / } \\
\text { Enfermeiros }\end{array}$ & $\mathrm{N} 2 * * *$ \\
\hline
\end{tabular}

* Corresponde a estudos primários com questões clínicas direcionadas para o tratamento/intervenção;

** Corresponde a estudos primários com questões clínicas direcionadas para o prognóstico ou a etiologia;

*** Corresponde a estudos primários com questões clínicas direcionadas para o significado ou a experiência.

Fonte: Autores (2020).

Após a leitura atentiva, foi possível caracterizar os resultados: fatores institucionais/laborais (as falhas e manutenção de equipamentos, materiais, gerenciamento, entre outros); fatores humanos (habilidades/conhecimentos, aspectos psicológicos e fisiológicos) (Harada, 2006), e ainda fatores relativos à gravidade do quadro clínico paciente assistido (Brasil, 2013). A Tabela 1 apresenta os fatores relacionados à intensificação do trabalho da enfermagem em âmbito hospitalar. 
Tabela 1 - Fatores relacionados com a intensificação do trabalho. Santa Maria - RS, 2020.

\begin{tabular}{|c|c|}
\hline & ITENS SELECIONADOS \\
\hline 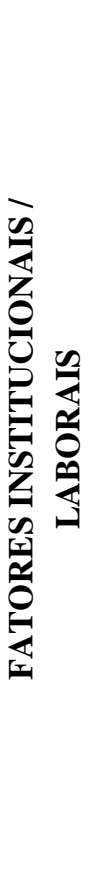 & $\begin{array}{l}\text { Indisponibilidade e/ou baixa qualidade de materiais e equipamentos A5; A13. } \\
\text { Sub dimensionamento de pessoal A3; A5; A11; A12, A20. } \\
\text { Precarização do trabalho A7. } \\
\text { Extensão da jornada de trabalho A17. } \\
\text { Realização de horas extras A17. } \\
\text { Duplicidade da jornada de trabalho A17. } \\
\text { Aumento nas horas de trabalho A17. } \\
\text { Manutenção dos vínculos empregatícios A14. } \\
\text { Precariedade nos contratos de trabalho A20. } \\
\text { Falta de controle sobre o tempo de trabalho A20. } \\
\text { Aumento da pressão no trabalho A20. } \\
\text { Menor nível de autonomia A1. } \\
\text { Menor flexibilidade nos horários de trabalho A1. } \\
\text { Menor participação na tomada de decisão A1. } \\
\text { Fragilidade nas questões de liderança e gerencia do enfermeiro A5. } \\
\text { Falta de apoio das gerências A11. } \\
\text { Fragilidade em relação à cultura de segurança do paciente A5. } \\
\text { Não reconhecimento da intensificação do trabalho do enfermeiro A19. } \\
\text { Aumento da carga laboral A6; A8. }\end{array}$ \\
\hline 选 & $\begin{array}{l}\text { Alterações físicas e emocionais A1; A16; A13. } \\
\text { Aumento do estresse A18. } \\
\text { Diminuição da satisfação no trabalho A8; A18. } \\
\text { Exaustão, despersonalização e clima negativo A15. } \\
\text { Fragilidade nas relações de trabalho A14. } \\
\text { Aumento do absenteísmo A10. } \\
\text { O trabalho como fonte de sofrimento A6. } \\
\text { Índice de estresse e baixo equilíbrio entre trabalho e vida pessoal A14. }\end{array}$ \\
\hline 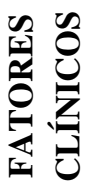 & $\begin{array}{l}\text { Aumento nas taxas de admissões a altas hospitalares A11. } \\
\text { Aumento nas taxas de mortalidade dos pacientes A4. } \\
\text { Tempo de permanência hospitalar A9. } \\
\text { Ocorrência de eventos adversos A2, A3, A9 A12. }\end{array}$ \\
\hline
\end{tabular}

Fonte: Autores (2020).

Conforme exposto na tabela acima, a intensificação do trabalho da enfermagem está relacionada aos fatores institucionais/laborais, fatores humanos e aqueles relativos ao quadro clínico dos pacientes. Esta intensificação vem sendo descrita como sendo uma das mudanças recentes impostas pelas novas formas de organização do trabalho da enfermagem nacional e internacionalmente.

\section{Discussão}

$\mathrm{Na}$ análise dos fatores institucionais/organizacionais observa-se a relação entre a intensificação do trabalho e a disponibilidade e qualidade de materiais e equipamentos ${ }^{(\mathrm{A} 5, \mathrm{~A} 13)}$ necessários para as atividades cotidianas nas instituições hospitalares. Estudo realizado com 309 enfermeiras atuantes em hospitais públicos e privados no Quênia, Tanzânia e Uganda, 
revela que estes profissionais experienciam condições de trabalho desfavoráveis, relacionadas à carência de materiais e equipamentos (Doef, et al., 2012). Situação também vivenciada em um hospital universitário da Coréia do Sul onde a assistência de enfermagem é diretamente influenciada pela falta de materiais e equipamentos (Kim, Yoo \& Seo, 2018).

Estudo realizado em 15 hospitais da região Nordeste do Brasil identificou que, para os enfermeiros, o item "condições de trabalho" contribuiu com 46,8\% para a explicação da precarização do trabalho. O mesmo estudo aponta que, para os técnicos de enfermagem, a intensidade do trabalho foi a dimensão que mais explicou o constructo da precarização com 52,29\% (Araújo-Dos-Santos et al., 2018). Assim, entende-se que a precarização do trabalho está intimamente ligada a intensificação do labor. Segundo Benfatti; Dantas, (2017), a intensificação e a precarização laboral são consideradas um traço característico do capitalismo e tem levado ao consumo desmedido das energias físicas e mentais dos colaboradores.

A precarização do trabalho também foi revelada por estudo no qual os problemas cruciais enfrentados por enfermeiros poloneses estão relacionados à falta de controle sobre o tempo de trabalho, às formas de contrato e ao aumento da pressão no trabalho (Kubisa, 2016). Também evidenciou-se que enfermeiros atuantes em instituições hospitalares apresentam menos probabilidade de ter autonomia, flexibilidade nos horários de trabalho e participação na tomada de decisão do que aqueles enfermeiros que atuam em outros locais de trabalho (Aeschbacher \& Addor, 2018). Assim, entende-se que é importante refletir sobre a atuação do enfermeiro nos diferentes espaços de cuidado, tendo em vista que a autonomia e a tomada de decisão são preceitos indispensáveis para a qualidade da assistência.

As condições de trabalho também foram citadas em estudo australiano, segundo o qual os enfermeiros do setor público relataram maior intensificação do trabalho em consequência da extensão da jornada, realização de horas extras, duplicidade da jornada, aumento nas horas de trabalho (Willis et al., 2008). Estudo canadense, realizado com 12 enfermeiros, aponta que o clima das relações de trabalho e a intensificação do trabalho acarretam em altos índices de estresse, afetando, diretamente, a manutenção dos vínculos empregatícios (Hart \& Warren, 2015).

Cabe inferir que condições de trabalho fragilizadas, implicam em maior índice de estresse nos trabalhadores, o que por sua vez, pode trazer prejuízos na qualidade da assistência ao paciente. Assim, a intensificação do trabalho está associada ao estresse, e este medeia à associação da intensificação do trabalho à satisfação intrínseca no trabalho (Sayin, et al,. 2018).

Deste modo, cabe destacar que a falta de liderança e gerência, por parte dos enfermeiros, podem influenciar para a diminuição da qualidade da assistência e segurança do paciente (Kim; Yoo; Seo, 2018). Nesta direção, estudo realizado na Nova Zelândia revela que os gerentes de enfermagem foram responsabilizados por evitar ou não reconhecer a intensificação do trabalho, o que influencia na qualidade da assistência (Willis, et al., 2007). Sobre isso, estudo realizado com enfermeiros australianos sugere que quando estes, são forçados a racionar os cuidados, prioriza-se as atividades clínicas, o que está relacionado a fragilidades nos processos gerenciais (Willis et al., 2015).

Os fatores relacionados à insatisfação no trabalho podem influenciar diretamente as atividades laborais do enfermeiro (A6, A8, A18). Estudo Etíope realizado com enfermeiros de hospitais públicos destaca que 33,5\% dos participantes apresentam baixo nível de satisfação no trabalho. As caracteristicas do trabalho nas unidades de internação estão negativamente associadas à satisfação do trabalho do enfermeiro, ao passo que há um aumento na carga de trabalho (Semachew et al., 2017). De acordo com estudo canadense, os enfermeiros percebem a intensificação do seu trabalho desde 1990, o que contribui para aumentar o estresse e diminuir a satisfação no trabalho (Zeytinoglu et al., 2007).

As atividades laborais também podem ser fonte de sofrimento para trabalhadores, por conviverem com a dor, o sofrimento e a morte de pacientes e repercutirem em intensificação do trabalho (Barboza, et al., 2018). As evidências destacam

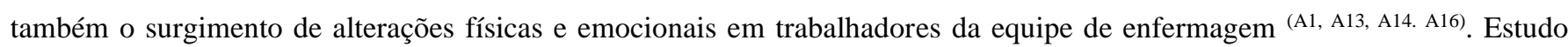


sueco revela que enfermeiros atuantes em hospitais públicos vêm apresentando crescentes cargas de trabalho ao longo de uma década. $\mathrm{O}$ trabalho foi intensificado, tanto em termos físicos quanto emocionais.

Outro estudo ${ }^{(\mathrm{A} 13)}$ destaca que, enfermeiros de hospitais públicos têm uma satisfação no trabalho menor do que as de hospitais particulares. O burnout está associado, principalmente, à alta carga de trabalho e fornecimento inadequado de informações, enquanto que as queixas somáticas estão associadas a condições físicas de trabalho exigentes (Doef, et al., 2012). Nesta direção, estudo canadense aponta a relação entre o clima das relações de trabalho e a intensificação do trabalho, o que gera um elevado índice de estresse e baixo equilíbrio entre trabalho e vida pessoal (Hart; Warren, 2015). Já estudo realizado em Barcelona aponta que as fragilidades nas condições de trabalho acarretam em menor satisfação, o que decorre principalmente pela exaustão, despersonalização e clima negativo (Granero, Blanch \& Ochoa, 2018).

Outro estudo realizado na região Centro-oeste do Brasil analisou as cargas de trabalho, processos de desgaste e absenteísmo por doença entre trabalhadores de enfermagem. As cargas fisiológicas representam $37 \%$ e cargas psíquicas correspondem a 36\%. As causas de afastamento estão relacionadas às doenças do sistema osteoconjuntivo e tecido muscular e os transtornos mentais e comportamentais. Essas notificações culminaram em absenteísmo de 1.567 dias, devido aos afastamentos para tratamento da doença (Mininel, et al., 2013).

As evidências relacionadas à gravidade do paciente apontam a relação da intensificação do trabalho com o aumento nas taxas de admissões a altas hospitalares ${ }^{\mathrm{A} 11}$, aumento nas taxas de mortalidade dos pacientes ${ }^{\mathrm{A} 4}$ e tempo de permanência hospitalar $^{\mathrm{A} 9}$. Também foram encontradas evidências da intensificação relacionada ao sub dimensionamento de pessoal (A3, A5, A11, A12, A20), ao aumento da carga de trabalho ${ }^{(\mathrm{A} 6, \mathrm{~A} 8)}$, à rotatividade de profissionais ${ }^{(\mathrm{A} 12)}$ nas unidades de internação e ao absenteísmo $^{(\mathrm{A} 10)}$. Tais evidências trazem implicações significativas para a efetividade do cuidado, dentre as quais se destacam: acréscimo das taxas de quedas do leito ${ }^{(\mathrm{A} 2, \mathrm{~A} 12)}$, infecções relacionadas a procedimentos invasivos ${ }^{(\mathrm{A} 12, \mathrm{~A} 9)}$, erros de medicação ${ }^{(\mathrm{A} 3 .}$ ${ }^{\mathrm{A} 12)}$, incidentes e eventos adversos decorrentes da prática do banho de leito ${ }^{(\mathrm{A} 12)}$, ao transporte de pacientes ${ }^{(\mathrm{A} 12)}$, e à satisfação dos pacientes com o cuidado de enfermagem ${ }^{(\mathrm{A} 9)}$.

Estudo realizado com enfermeiros australianos aponta que, a intensificação do trabalho esteve relacionada com o aumento nas taxas de admissões a altas nas unidades de internação (Willis et al., 2015). Acredita-se que os procedimentos de internação e alta dos pacientes demandam mais cuidados como: a identificação do paciente, encaminhamento para o quarto/leito, punção de acesso venoso, aprazamento da prescrição médica e preparo e administração de medicamentos, e atendimento das demais necessidades do paciente. Em relação aos cuidados específicos do enfermeiro, entende-se que este precisa realizar o histórico de enfermagem, juntamente com as demais etapas da Sistematização da Assistência de Enfermagem (SAE), aliando a outros procedimentos assistenciais e gerenciais, de acordo com as demandas do paciente.

Estudo realizado em quatro hospitais da Finlândia revela uma associação entre aumento da carga de trabalho diária do enfermeiro e ocorrência de incidentes e eventos adversos. Este aumento da carga de trabalho pode elevar os índices de incidentes entre $8 \%$ e 34\%, mostrando um acréscimo de $40 \%$ das chances do paciente ir a óbito (Fagerström, Kinnunen \& Saarela, 2018). Em estudo brasileiro evidenciou-se associação entre carga de trabalho nas unidades de internação e os resultados referentes à média de permanência hospitalar (Magalhães et al., 2017). Dados semelhantes também foram encontrados em estudo realizado na região Sudeste do Brasil, apontando associação entre incidentes e tempo de permanência do paciente em unidade de terapia intensiva de trauma. Os autores trazem que os incidentes sem dano tiveram associação com a carga de trabalho de enfermagem (Padilha et al., 2017).

Em estudo de Freitas et al., (2019), que avaliou a carga de trabalho em terapia intensiva, evidenciou-se, que esta foi maior no primeiro e no último dia de internação. Os autores destacam que isso ocorre pelas demandas especificas da admissão e alta do paciente. O estudo também aponta que a carga de trabalho esteve aumentada em pacientes com maior nível de 
gravidade e que evoluíram para óbito. Neste contexto, evidencia-se na literatura cientifica uma série de fatores que se relacionam com intensificação do trabalho da enfermagem no ambiente hospitalar. No entanto, não há estudos que discutem claramente essa relação ou que mensurem esse constructo.

\section{Considerações Finais}

O presente estudo possibilitou a identificação dos fatores relacionados à intensificação do trabalho da enfermagem a nível hospitalar. As evidências apontam a ocorrência de fatores institucionais/laborais, fatores humanos e fatores clínicos. Em relação aos primeiros, evidencia-se que estão atrelados à precarização do trabalho, sub dimensionamento de pessoal com consequente aumento da carga de trabalho, fragilidades nas condições e relações de trabalho, extensão e/ou duplicidade na jornada de trabalho e, neste interim, a realização de varias tarefas de modo simultâneo. Já em relação aos fatores humanos, evidencia-se a ocorrência de alterações físicas e mentais em trabalhadores, resultando em aumento do estresse e diminuição da satisfação no trabalho, o que repercute no aumento do absenteísmo e baixo equilíbrio entre trabalho e vida pessoal.

As evidências também apontam para os fatores clínicos, havendo relação direta da intensificação do trabalho com o aumento nas taxas de admissões a altas hospitalares, aumento nas taxas de mortalidade dos pacientes e tempo de permanência hospitalar. Tais evidências trazem implicações significativas para a efetividade do cuidado, dentre as quais se destacam: acréscimo das taxas de quedas do leito, infecções relacionadas a procedimentos invasivos, erros de medicação, incidentes e eventos adversos decorrentes da prática do banho de leito, ao transporte de pacientes, e à satisfação dos pacientes com o cuidado de enfermagem.

Como potencialidade o estudo traz contribuições no sentido de identificar os fatores que se relacionam com a intensificação do trabalho da enfermagem. Possíveis aspectos limitantes neste estudo estão relacionados ao nível II e VI, quanto à força da evidência. A maioria dos estudos analisados apresenta a questão clínica direcionada para o prognóstico e/ou etiologia. Sugere-se que novos estudos possam ser realizados subsidiando a formulação de estratégias e políticas direcionadas a melhoria das condições de trabalho desta categoria profissional.

\section{Agradecimentos}

À Universidade de Cruz Alta - Unicruz, pelo apoio e incentivo no processo de doutoramento do primeiro autor, através do Programa Institucional de Capacitação Docente (PICD).

Ao Grupo de Estudos em “Trabalho, Enfermagem, Saúde, Segurança e Meio Ambiente” - GETESSMA, vinculado ao PPGEnf/UFSM, pelos conhecimentos compartilhados e pelas vivências enriquecedoras.

\section{Referências}

Aeschbacher, R., \& Ddor, V. (2018). Institutional effects on nurses' working conditions: a multi-group comparison of public and private non-profit and forprofit healthcare employers in Switzerland. Human Resources for Health, 16(58),1-10.

Aires, K. F. et al. (2016). Contribution of the work load to the occurrence of medication errors in nursing. Rev. Enferm. UFPE on line. 10(12)4572-80.

Araújo-Dos-Santos, T. et al. (2018). Job insecurity among nurses, nursing technicians and nursing aides in public hospitals. Rev Esc Enferm USP. 52,e03411. 2018 .

Barboza, P. C. et al. (2018). The meaning of work: perspectives of nursing professionals who work in clinical units. Rev Rene. 19 , e32819.

Brasil, Agência Nacional de Vigilância Sanitária. Manual Segurança do Paciente e Qualidade em Serviços de Saúde: uma reflexão teórica aplicada à prática. Brasília: ANVISA, 2013.

Bunner, J., Prem, R., \& Korunka, C. (2018). How Work Intensification Relates to Organization-Level Safety Performance: The Mediating Roles of Safety Climate, Safety Motivation, and Safety Knowledge. Frontiers in Psychology. 9, 1-13. 
Carlesi, K. C. et al. (2017). Patient Safety Incidents and Nursing Workload. Rev. Latino-Am. Enfermagem. 25 , e2841.

Coutinho, D. C. M., Alves, G. F., \& Santos, R. (2020). Trabalho e desemprego no capitalismo: reflexões para o Serviço Social. O social em Questão, 47, 129150 .

DOEF, M. V. D., Mbazzi, F. B., \& Verhoeven, C. (2012). Job conditions, job satisfaction, somatic complaints and burnout among East African nurses. Journal of Clinical Nursing. 21, 1763-1775.

Fagerström, L., kinnunen, M., \& Saarela, J. (2018). Nursing workload, patient safety incidents and mortality: an observational study from Finland. BMJ Open, $8, \mathrm{e} 016367$.

Fineout-Overholt, E. \& Stillwell, S. B. (2011). Asking compelling, clinical questions. In: Melnyk, B. M., Fineout-Overholt, E., editors. Evidence-based practice in nursing \& healthcare: a guide to best practice. Philadelphia: Wolters Kluwer Health/Lippincott Williams \& Wilkins, 25-39.

Forte, E. C. N. et al. (2019). Work process: a basis for understanding nursing errors. Revista da Escola de Enfermagem da USP, 53 , e03489.

Francisco, E. M. V. (2020). A precarização das relações e condições de trabalho dos(as) assistentes sociais em tempos de "reforma trabalhista". O Social em Questão, 47, 65-84.

Freitas, E. O. et al. (2019). Carga de trabalho da enfermagem em unidade de terapia intensiva: um estudo de método misto. Research, Society and Development, 9(1), e161911733.

Granero, A., Blanch, J., Ochoa, P. (2018). Labor conditions and the meanings of nursing work in Barcelona. Rev. Latino-Am. Enferm. 26 , e2947.

Harada, M. J. C. S. (2006). A prevenção do erro humano. In: Harada, Maria de Jesus Castro Sousa, Pedreira, Mavilde da Luz Gonçalves Organizadores. O erro humano e a segurança do paciente. São Paulo: Atheneu, 2006.

Hart, S. M., \& Warren, A. M. (2015). Understanding nurses' work: Exploring the links between changing work, labour relations, workload, stress, retention and recruitment. Economic and Industrial Democracy. 36(2)305-329.

Hopia, H., Latvala, E., \& Liimatainen, L. (2016). Reviewing the methodology of an integrative review. Scand J Caring Sci, 30(4)662-9.

Kim, K., Yoo, M. S. S., \& Seo, E. J. (2018) Exploring the Influence of Nursing Work Environment and Patient Safety Culture on Missed Nursing Care in Korea. Asian Nursing Research, 12, 21-126.

Kubicek, B., Paškvan, M., \& Korunka, C. (2015). Development and validation of an instrument for assessing job demands arising from accelerated change: The intensification of job demands scale (IDS). European Journal of Work and Organizational Psychology, 24(6)899-913.

Kubisa, J. From better job quality to higher-quality care - Polish nurses' collective struggle with the public healthcare system. Emecon, 6(1)1-14.

Magalhães, A. M. M., et al. (2017). Association between workload of the nursing staff and patient safety outcomes. Rev Esc Enferm USP, 51 , e03255.

Magalhães, A. M. M., et al. (2013). Nursing workload and patient safety - a mixed method study with an ecological restorative approach. Rev. Latino-Am. Enferm, 21(spe)46-154.

Marx, K. (2013). O capital: crítica à economia política. Livro I: O processo de produção do capital. (31a ed.), Civilização Brasileira.

Mendes, K. D. S., Silveira, R. N. C. P., \& Galvão, C. M. (2019). Use of the bibliographic reference manager in the selection of primary studies in integrative reviews. Texto contexto - enferm. 28, e20170204.

Mininel, V. A. et al. (2013). Workloads, strain processes and sickness absenteeism in nursing. Rev. Latino-Am. Enferm, 21(6)290-7.

Moher, D. et al. (2009). Preferred reporting items for systematic reviews and meta-analyses: the PRISMA statement. Int J Surg, 6(7), e1000097.

Pina, J. A. \& Stotz, E. N. (2014). Intensificação do trabalho e saúde do trabalhador: uma abordagem teórica. Rev. bras. Saúde ocup, 39(130) 150-160.

Pires, D. E. P. (2008). Reestruturação produtiva e trabalho em saúde no Brasil. (2a ed.), Confederação Nacional dos Trabalhadores em Seguridade Social, Annablume.

Pochmann, M. (2020). Tendências estruturais no mundo do trabalho no Brasil. Ciênc. saúde coletiva, 25(1)89-99.

Rocha, T. A. (2020). O culto da performance: as percepções do trabalho contemporâneo e a saúde mental dos trabalhadores na cidade de Araraquara - RS. Dissertação (Mestrado) - Programa de Pós-Graduação da Faculdade de Ciências e Letras - UNESP/Araraquara. Araraquara, São Paulo.

Sayin, F, K. et al. (2018). The role of work intensification in intention to stay: A study of personal support workers in home and community care in Ontario, Canada. Economic and Industrial Democracy, 1-20.

Selberg, R. (2013). Nursing in times of neoliberal change: an ethnographic study of nurses' experiences of work intensification. Nordic journal of working life studies, 3(2)9-35.

Semachew, A. et al. (2017) Predictors of job satisfaction among nurses working in Ethiopian public hospitals, 2014: institution-based cross-sectional study. Human Resources for Health, 15(31)1-8.

Souza, N. V. D. O. et al. (2017). Neoliberalist influences on nursing hospital work process and organization. Rev Bras Enferm, 70(5) 912-9. 
Research, Society and Development, v. 10, n. 1, e22210111703, 2021

(CC BY 4.0) | ISSN 2525-3409 | DOI: http://dx.doi.org/10.33448/rsd-v10i1.11703

Stillwell, S. B et al. (2010). Searching for the Evidence Strategies to help you conduct a successful search. AJN, 10(5)41-47.

The Joanna Briggs Institute. (2014). Joanna Briggs Institute Reviewers' Manual: 2014 edition. [Internet] The Joanna Briggs Institute. http://joannabriggs.org/assets/docs/sumari/reviewersmanual-2014.pdf.

Willis, E. et al. (2017). Work Intensification and Quality Assurance Missed Nursing Care. J Nurs Care Qual, 1-7.

Willis, E. et al. (2015). Work intensification as missed care. Labour and Industry, 25(2)118-133.

Willis, E. et al. (2008). Enterprise bargaining: a case study in the de-intensification of nursing work in Australia. Nursing Inquiry, 15(2) 148-157.

Zeytinoglu, I. U. et al. (2007). Associations between Work Intensification, Stress and Job Satisfaction: The Case of Nurses in Ontario. Relations industrielles / Industrial Relations, 62(2)201-225. 